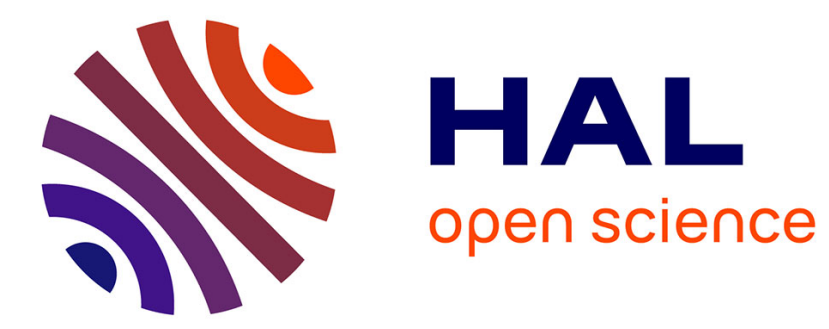

\title{
Navigating at Will on the Water Phase Diagram
}

S. Pipolo, M. Salanne, G. Ferlat, S. Klotz, A. m. M Saitta, F. Pietrucci

\section{To cite this version:}

S. Pipolo, M. Salanne, G. Ferlat, S. Klotz, A.m. M Saitta, et al.. Navigating at Will on the Water Phase Diagram. Physical Review Letters, 2017, 119 (24), 10.1103/physrevlett.119.245701 . hal01921261

\section{HAL Id: hal-01921261 \\ https: / hal.sorbonne-universite.fr/hal-01921261}

Submitted on 27 Nov 2018

HAL is a multi-disciplinary open access archive for the deposit and dissemination of scientific research documents, whether they are published or not. The documents may come from teaching and research institutions in France or abroad, or from public or private research centers.
L'archive ouverte pluridisciplinaire HAL, est destinée au dépôt et à la diffusion de documents scientifiques de niveau recherche, publiés ou non, émanant des établissements d'enseignement et de recherche français ou étrangers, des laboratoires publics ou privés. 


\title{
Navigating at Will on the Water Phase Diagram
}

\author{
S. Pipolo, ${ }^{1, *}$ M. Salanne ${ }^{2}$ G. Ferlat,${ }^{1}$ S. Klotz, ${ }^{1}$ A. M. Saitta, ${ }^{1}$ and F. Pietrucci ${ }^{1, *}$ \\ ${ }^{1}$ Sorbonne Universités, UPMC Université Paris 06, CNRS UMR 7590, IRD UMR 206, MNHN, IMPMC, F-75005 Paris, France \\ ${ }^{2}$ Sorbonne Universités, UPMC Université Paris 06, CNRS, Laboratoire PHENIX, F-75005 Paris, France \\ (Received 21 June 2017; revised manuscript received 21 September 2017; published 14 December 2017)
}

\begin{abstract}
Despite the simplicity of its molecular unit, water is a challenging system because of its uniquely rich polymorphism and predicted but yet unconfirmed features. Introducing a novel space of generalized coordinates that capture changes in the topology of the interatomic network, we are able to systematically track transitions among liquid, amorphous, and crystalline forms throughout the whole phase diagram of water, including the nucleation of crystals above and below the melting point. Our approach, based on molecular dynamics and enhanced sampling or free energy calculation techniques, is not specific to water and could be applied to very different structural phase transitions, paving the way towards the prediction of kinetic routes connecting polymorphic structures in a range of materials.
\end{abstract}

DOI: 10.1103/PhysRevLett.119.245701

Computational structure prediction methods $[1,2]$ have strongly contributed to the rapid increase of new predicted phases of materials with enhanced properties for applications (see, e.g., Ref. [3]). However, at present, no general approach has been developed for guiding experiments through the pathways connecting stable structures of condensed matter. Moreover, metastable phases are very often involved in phase transitions [4] and sometimes their kinetic stability is very high [5]. Thus, in order to recover the global minimum structure, one needs to find specific routes, by, e.g., acting on pressure or temperature, in a way that is not at all trivial to guess [6]. A precise understanding of transition mechanisms and the corresponding kinetics is therefore the key to explain and control the behavior of matter. The case of water is emblematic because several experiments have disclosed connections between stable and metastable phases [7-10] and recently simulations have highlighted the importance of metastable states in understanding the mechanism of phase transitions and related transformations [11]. A classic example is the connection between the crystalline ice stable at ambient pressure (Ice I), and the low-density amorphous (LDA) and high-density amorphous (HDA) ices: by compressing Ice I up to $10 \mathrm{kbar}$ at $\approx 80 \mathrm{~K}$ one obtains HDA ice instead of Ice VI [12], which may be transformed into LDA ice by decompression of HDA ice at $130 \mathrm{~K}$ [10] or by heating recovered HDA ice at ambient pressure to beyond $130 \mathrm{~K}$ [9]; finally Ice I is recovered by heating up LDA ice. Similar connections between crystalline and amorphous ices are found in the high-pressure region of the water phase diagram where a very-high-density amorphous (VHDA) ice, plastic ices, and crystalline structures with a complex hydrogen-bond network (e.g., Ice VII) have been observed or predicted $[5,13]$.

Molecular dynamics (MD), a simulation method that yields the atomic trajectories as a function of time at given thermodynamic conditions, is in principle able to track such transitions. The kinetic barriers are, however, generally too large to allow an efficient exploration of the configuration space within typical MD timescales. Hence, so far it has been necessary to introduce (i) simplistic interaction models [14] and/or (ii) seeding techniques [15]. Another approach consists in using enhanced sampling techniques that accelerate the occurrence of rare events by focusing on low-dimensional order parameters, also called collective variables (CVs) [16]. Yet the CVs available to describe phase transitions are specifically designed for a given type of structural transformation [17-19], while no general CV scheme has been proven successful for a wide class of problems, in particular those involving amorphous systems. Recently, distance metrics developed for condensed matter [20-25] have been proven to be successful in classifying structures in molecular or extended systems based on their atomic environment and/or interatomic network. Here we show that by combining enhanced sampling techniques with a novel CV based on a general metric we are able to define in an efficient way the topological space of transformations among liquid, amorphous, and crystalline forms of water. This allows exploring at will the phase diagram along pathways connecting minimum-energy structures with a single, general approach, capable to characterize mechanisms and energetics.

Our CV scheme relies on the concept of generalized distances between configurations of the system, as defined from relative atomic positions, and it only requires to postulate the initial and final states of the target transformation, without any assumption on the pathway. The transformation is represented in a two-dimensional space $\{s, z\}$ of path CVs [26], with $s$ quantifying the progress of the transformation and $z$ allowing us to discriminate between different pathways and to represent transitions to states that are not the target ones. In such a CV scheme each configuration of the system is associated with a permutation invariant vector [21] (PIV), built up from 
interatomic Cartesian distances. The PIV is built starting from atom-type-specific ordered blocks, $\mathbf{v}_{k k^{\prime}}$, with elements

$$
v_{k k^{\prime}}^{\beta \beta^{\prime}}=c_{k k^{\prime}} \mathcal{S}\left(\sqrt[3]{\frac{\Omega_{0}}{\Omega}}\left|\mathbf{r}_{\beta k}-\mathbf{r}_{\beta^{\prime} k^{\prime}}\right|\right)
$$

Here $\mathbf{r}_{\beta k}$ is the position vector of the $\beta$ th atom of type $k$ (oxygen or hydrogen), with $\beta>\beta^{\prime}, k>k^{\prime} ; c_{k k^{\prime}}$ are coefficients that define the PIV variant (they are all equal to one in the original formulation); $\Omega$ and $\Omega_{0}$ are the volume of the simulation box and a reference volume (details below), respectively; $\mathcal{S}$ is a switching function monotonically decreasing from one to zero as $\left|\mathbf{r}_{\beta k}-\mathbf{r}_{\beta^{\prime} k^{\prime}}\right|$ increases (see Supplemental Material Ref. [27] for more details). In order to define the PIV, first the $v_{k k^{\prime}}^{\beta \beta^{\prime}}$ elements of each $\mathbf{v}_{k k^{\prime}}$ block are sorted in ascending ordered, then the different blocks are simply joined together resulting in a PIV of $N_{\text {atoms }}\left(N_{\text {atoms }}-1\right) / 2$ components, that we indicate with $V_{\alpha}$. The sorting operation within each block introduces invariance upon permutation of identical atoms. The volume scaling factor (absent in Ref. [21]) was found to be important to avoid violent fluctuations of the cell parameters during metadynamics.

Distances between generic configurations $X$ and $Y$ are computed as squared Euclidean distances between the corresponding PIVs $\left(\mathcal{D}_{Y X}=\sum_{\alpha}\left(V_{Y \alpha}-V_{X \alpha}\right)^{2}\right)$, and used to map each configuration of the system $(X)$ into a point $\left(s_{X}, z_{X}\right)$ in the 2D space defined by the path CVs [26], built starting from only two reference configurations $A$ and $B$ representing the initial and final state of the transformation:

$$
\begin{aligned}
& s_{X}=\frac{1 \times e^{-\lambda \mathcal{D}_{A X}}+2 \times e^{-\lambda \mathcal{D}_{B X}}}{e^{-\lambda \mathcal{D}_{A X}}+e^{-\lambda \mathcal{D}_{B X}}} \\
& z_{X}=-\lambda^{-1} \log \left(e^{-\lambda \mathcal{D}_{A X}}+e^{-\lambda \mathcal{D}_{B X}}\right) .
\end{aligned}
$$

The two coordinates track the progress from $A$ to $B$ and the distance from $A$ and $B$, respectively. We remark that this formulation does not contain any guess about the mechanism of the transformation, and that the freedom granted by the $z$ coordinate allows us to explore also the formation of unexpected metastable structures different from $A$ and $B$ [e.g., Ice VII-P in Fig. 4(c)]. There is some freedom in the choice of the parameter $\lambda$ : in this work we adopted $\lambda \simeq 2.3 / \mathcal{D}_{A B}$, conveniently localizing the free energy basins of reference states $A$ and $B$ around $s \simeq 1.1$ and $s \simeq 1.9$, respectively, and leading to smooth transformation pathways and landscapes. A much larger $\lambda$ would produce very irregular and discontinuous pathways, while a much smaller one would hamper the resolution of different phases. We used metadynamics [38] for the discovery of continuum pathways between locally stable configurations, and umbrella sampling [39] for the reconstruction of precise free energy landscapes. Computational details are given in Ref. [27].
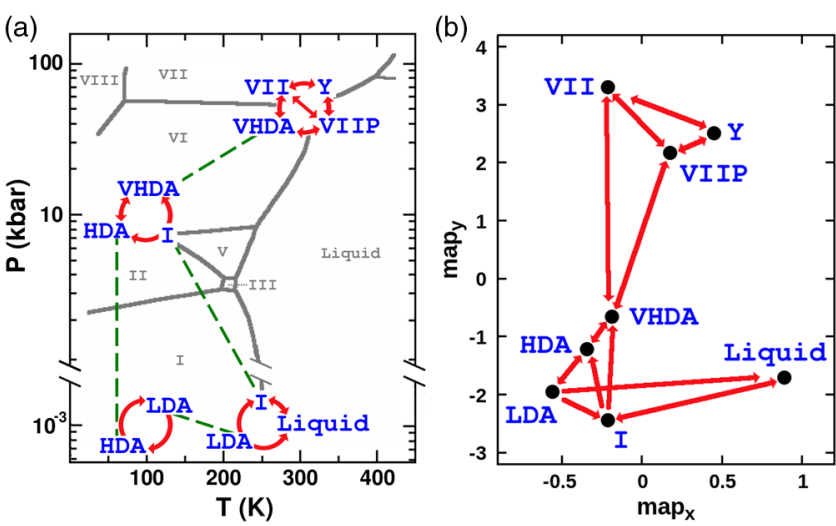

FIG. 1. (a) The TIP4P/2005 water phase diagram [40] is shown in grey, and phase transitions between (meta)stable phases (blue labels) simulated with metadynamics are indicated with red arrows. Dashed green lines represent variations of the $(\mathrm{P}, \mathrm{T})$ conditions of the system within a phase, performed with unbiased molecular dynamics simulations. (b) Two-dimensional map reproducing distances between PIV vectors defined in Eq. (1). The map axes are defined within an arbitrary rotation.

In Fig. 1(a) we draw the pathways we followed on the phase diagram of a realistic model of water [40], navigating within and across free-energy basins using standard MD and enhanced sampling techniques, respectively. Figure 1(b) shows a two-dimensional map (see also Ref. [22]) of distances between the visited crystalline and amorphous structures: the metric employed to define the CVs is able to scatter the different phases in a way that recalls the topology of the phase diagram, representing kinetically connected phases as neighbors, and kinetically disconnected ones as far apart.

As a starting point we analyze the crystallization of Ice I both from the liquid and the LDA phases at $P=1$ bar and over a range of temperatures around the melting point ( $T_{m} \simeq 250 \mathrm{~K}$ for the adopted interatomic potential [40]). The initial configurations have been respectively obtained by cooling down an equilibrium liquid phase from $T=$ $300 \mathrm{~K}$ and heating up a LDA structure from $T=100 \mathrm{~K}$ [27]. Both crystallization transitions have been achieved multiple times in metadynamics simulations at $T=240 \mathrm{~K}$ and $T=260 \mathrm{~K}$ and they are all characterized by (i) a nucleation mechanism that we show in Fig. 2 for the LDAIce I transformation at $T=240 \mathrm{~K}$, (ii) the formation of a crystal nucleus of cubic symmetry (Ice Ic), and (iii) a final state with either a perfect cubic symmetry or made up of layers of cubic Ic and hexagonal Ih ice [see last snapshot in Fig. 2(a)]. This last feature is in agreement with experimental findings [41], and our results show that the formation of stacking disordered Ice I may also proceed via the merging of Ic-nuclei as well as via (i) random growth of Ic and Ih layers [42] and (ii) direct formation of Ic-Ih nuclei [19]. In Fig. 2(b) we display the free energy profiles for the liquid-Ice I and LDA-Ice I transformations, respectively, above and below the melting point. The 

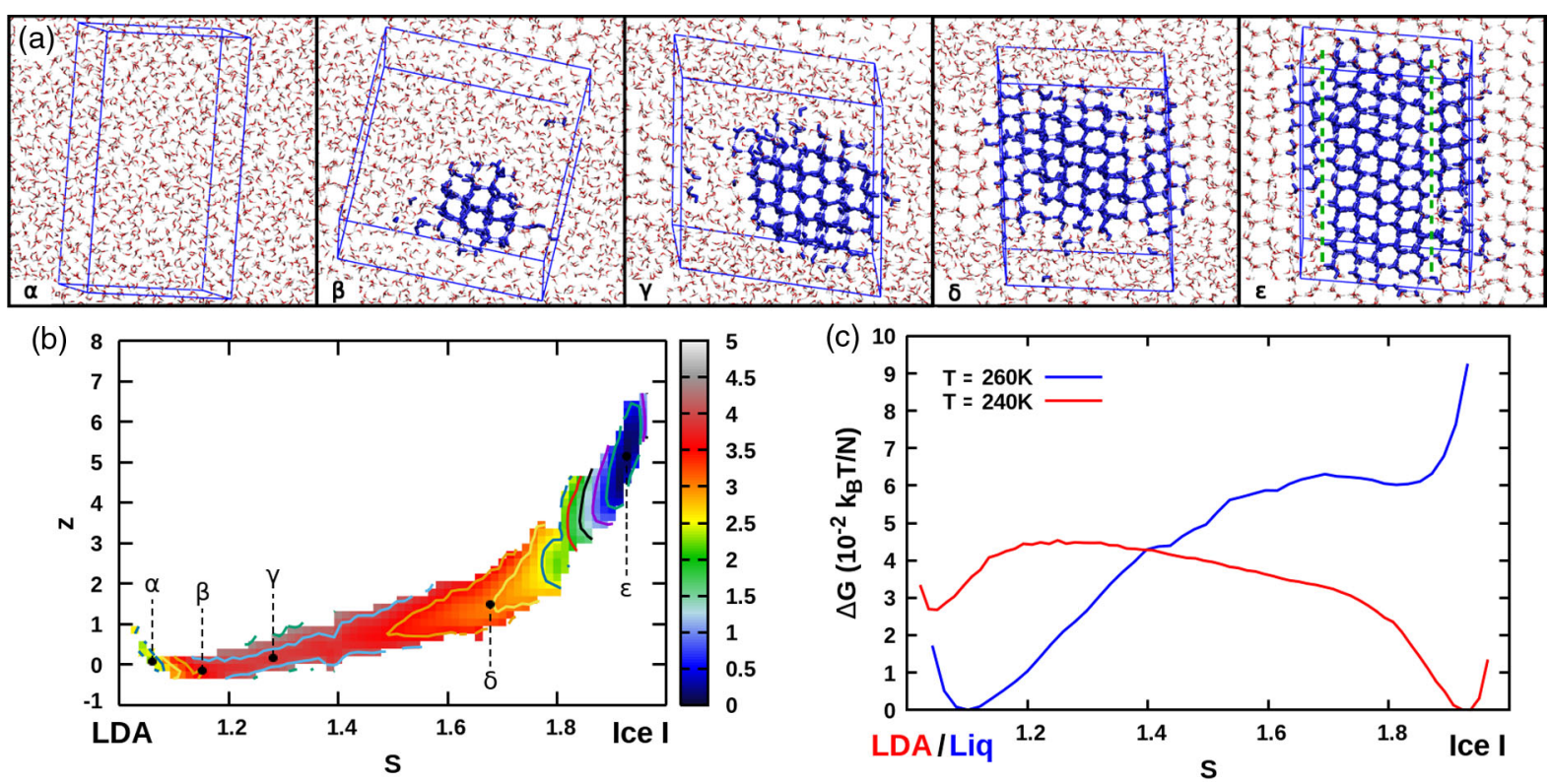

FIG. 2. (a) Sequence of snapshots $(\alpha, \beta, \gamma, \delta, \epsilon)$ of the umbrella sampling simulation describing the progressive crystallization of LDA water into Ice I. Molecules in icelike environment, with an average local tetrahedral bond order parameter [17] of oxygen higher than 0.7, are shown in blue (in the box only). Snapshot $\gamma$ shows the Ice I nucleus at its critical size (saddle point in the free-energy profile). Snapshot $\epsilon$ shows stacking disordered ice I, with Ic cubic regions separated by Ih hexagonal layers (green lines). (b) Free-energy profile along the LDA-Ice I transformation pathway $(T=240 \mathrm{~K}, P=1$ bar) obtained via the weighted histogram analysis method applied to umbrella sampling trajectories. (c) Comparison of the free-energy profiles, projected along the $s$ coordinate, for the crystallization transitions LDA-Ice I at $T=240 \mathrm{~K}$ and $P=1$ bar and liquid-Ice I at $T=260 \mathrm{~K}$ and $P=1$ bar. The free energy minima of each simulation are arbitrarily set to zero ( $N$ is the number of water molecules, here 800$)$.

calculated relative stabilities of the various phases agree with the phase diagram of the water model, and the order of magnitude of the free-energy profiles is consistent with free-energy differences calculated in previous works [43]. We remark that crystallizing the liquid above the melting temperature, in the bulk, without any seeds and with a very realistic water model, shows that our approach allows us to perform very challenging transformations even in unfavorable conditions, reaching metastable states (here Ice I) starting from the global minimum.

In order to carry on our continuous journey towards high pressure, we follow the experimental routes by cooling down Ice I to $T=100 \mathrm{~K}$, and then compressing it at $P=$ 10 kbar using standard MD: in the neighborhood of this point of the phase diagram we explore (with enhanced sampling simulations) the transformation to HDA ice as a function of temperature and pressure. We find that the freeenergy barrier decreases when temperature and pressure increase, as we show in Fig. 3. Furthermore we note that although the free energy of Ice I is higher than the one of HDA ice already at $P=10 \mathrm{kbar}$ and $T=100 \mathrm{~K}$ (which is expected since Ice I is not the stable phase in this region of the phase diagram), the free-energy barrier is nonzero. This result is in accordance with a common interpretation of the Ice I-HDA transformation $[9,44]$ that can be seen as an extrapolation to low temperatures and high pressures of the Ice I-liquid coexistence line. Even if this description is mostly qualitative, it certainly invigorates the idea that lowdensity crystalline ice is unstable with respect to denser disordered forms at high pressures, and that such instability occurs at higher pressures as temperature is decreased.

The HDA phase we obtain is then (i) decompressed at ambient pressure and transformed into LDA ice to close the loop of transitions at low pressure, (ii) compressed at $P=$ 12 kbar and transformed into VHDA ice. Free-energy and density profiles for HDA-LDA and HDA-VHDA transformations are provided [27]. The HDA-VHDA transformation connects the low-pressure and high-pressure regions that we explore in this work. The VHDA phase is compressed and heated until it reaches $P=50 \mathrm{kbar}$ and $T=300 \mathrm{~K}$, and at this point we address its crystallization to Ice VII. While simulating the VHDA-Ice VII transformation via metadynamics we observe that the system visits two additional metastable configurations. This result demonstrates that our method does not constrain the system to sample configurations along a simple path connecting the two reference structures, but rather allows it to follow complex mechanisms and discover new free energy basins. The metastable structures differ markedly from the other phases, as shown in Fig. 4 where we compare representative snapshots and the oxygen-oxygen radial distribution functions. The first metastable phase is identified as the plastic Ice VII-P, which had already been proposed by Himoto et al. [5]. Oxygen atoms are arranged in a rather 


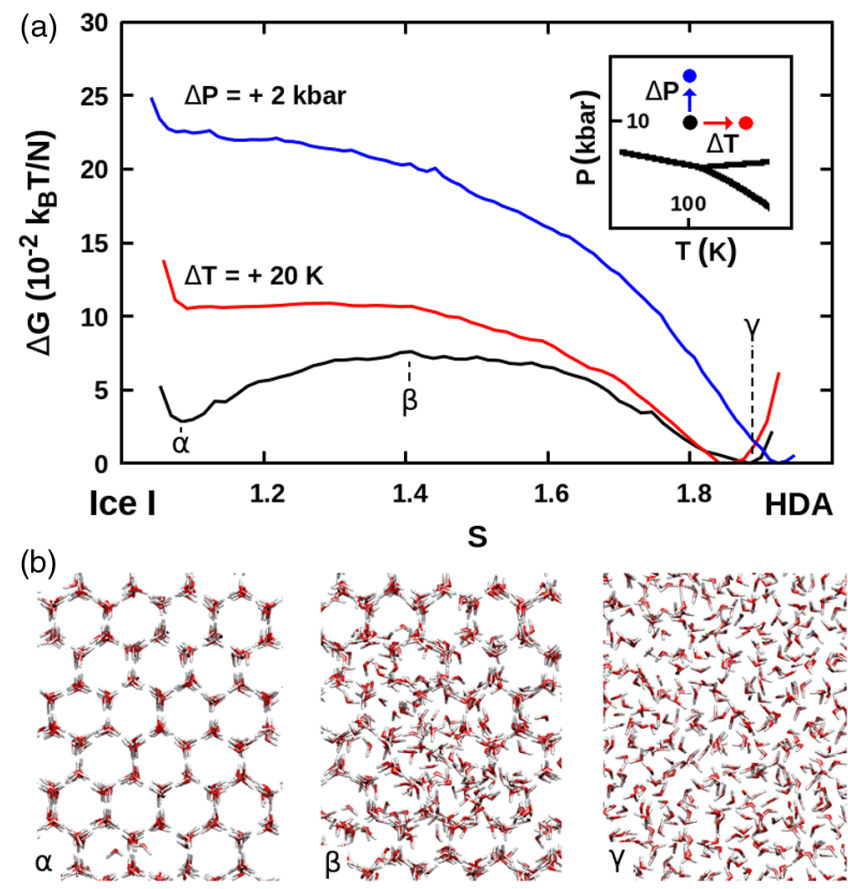

FIG. 3. (a) Free-energy profiles projected along the path collective variable $s$ for the Ice I-HDA transformation at different thermodynamic conditions: $T=100 \mathrm{~K}$ and $P=10$ kbar (black), $T=120 \mathrm{~K}$ and $P=10 \mathrm{kbar}$ (red), $T=100 \mathrm{~K}$ and $P=12 \mathrm{kbar}$ (blue). The free energy of HDA ice is arbitrarily set to zero. The inset shows the position of the three transformations in the phase diagram: at $T=100 \mathrm{~K}$ and $P=10 \mathrm{kbar}$ Ice I is metastable [see Fig. 1(a)] and separated from HDA ice by a barrier. (b) Sequence of snapshots $(\alpha, \beta, \gamma)$ along the amorphization process at $T=100 \mathrm{~K}$ and $P=10$ kbar. They are taken from the umbrella sampling trajectories and their position along the $s$ coordinate is shown in panel (a). ordered crystalline network, whereas the hydrogen bond network changes dynamically: the correlation decay time of molecular dipoles is more than one order of magnitude shorter than that of Ice VII at the same thermodynamic conditions. The second metastable phase (labeled here "Ice $Y$ ") is characterized by a tetragonal oxygen lattice and stacked layers of hydrogen-bond networks. We leave a detailed investigation of this phase for future works (the atomic coordinates are provided [27]). The flexibility of our method in representing transformations among several states in a two-dimensional CV space is illustrated in the free energy landscape connecting the three crystalline phases (Ice VII, Ice VII-P, and Ice Y) shown in Fig. 4(c).

In conclusion, we propose a versatile method allowing the efficient simulation of phase transitions in condensed matter. We illustrated the approach by tackling the difficult problem of poly(a)morphism in water, including kinetically challenging amorphous-to-crystalline and liquid-tocrystalline transitions. In particular, we simulated transformations between the LDA, HDA, liquid, and Ice I phases in the low-pressure region of the phase diagram and among VHDA ice and Ice VII in the high-pressure region. In both cases, important mechanistic information could be extracted from the simulations, highlighting the role of metastable structures during phase transitions. Thanks to a very general formulation, the proposed approach is not restricted to specific transitions of a single material. Analysis of 50 experimental polymorphs belonging to 13 different materials (covalent, metallic, ionic, and molecular) indicates that the PIV-based metric is able to resolve all physically distinct structures [27], suggesting a broad applicability of our simulation approach. The ability
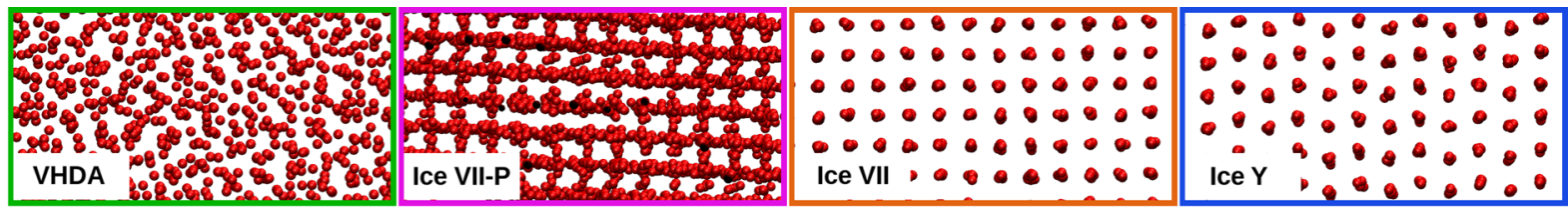

(a)
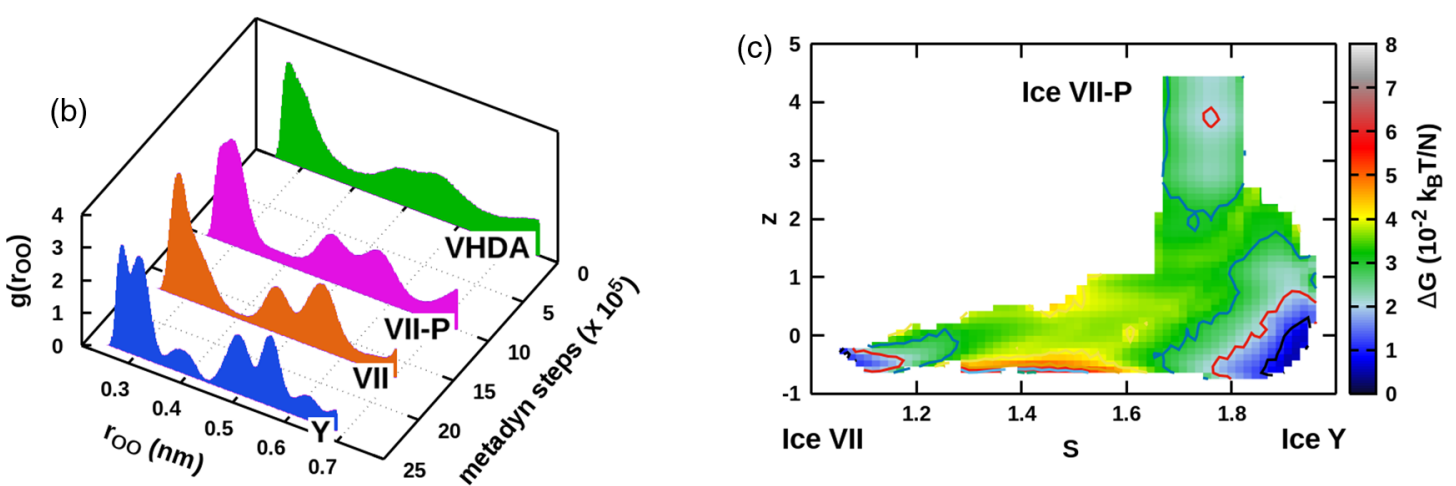

FIG. 4. (a) Snapshots representing the oxygen atoms of the system in four different configurations: VHDA, Ice VII-P (plastic phase), Ice VII, and Ice Y. All these structures are visited by the system while simulating the VHDA-Ice VII transformation at $T=300 \mathrm{~K}$ and $P=50$ kbar via metadynamics. (b) Oxygen-oxygen radial distribution functions as a function of the number of metadynamics simulation steps, for the stable configurations shown in panel (a). The color code is consistent with panel (a). (c) Free energy landscape at $T=300 \mathrm{~K}$ and $P=50 \mathrm{kbar}$ in the $\{s, z\}$ space obtained from umbrella sampling simulations. Equilibrated Ice VII and Ice $\mathrm{Y}$ are used to define the path collective variables. The simulation box includes $N=360$ water molecules. 
to discover transformation mechanisms, simulate nucleation events, and reconstruct free energy landscapes and kinetic barriers - all in a robust and systematic way-is highly complementary to structure prediction and materials discovery efforts.

F. P. thanks Grégoire A. Gallet for help with the implementation of a preliminary version of the PIV path collective variables. This work was funded within the Investissements d'Avenir program under reference ANR11-IDEX-0004-02, within the framework of the cluster of excellence MATériaux Interfaces Surfaces Environnement (MATISSE) led by Sorbonne Universités. We acknowledge calculations performed on the Gnome cluster at UPMC, on the Occigen cluster at CINES, Montpellier, France, and on the Ada cluster at IDRIS, Orsay, France, under GENCI allocations 2015-091387 and 2016-091387.

*silvio.pipolo@univ-lille.fr

Present address: Université de Lille, CNRS, Centrale Lille, ENSCL, Université d'Artois, UMR 8181-UCCS-Unité de Catalyse et Chimie du Solide, F-59000 Lille, France. †fabio.pietrucci@upmc.fr

[1] C. J. Pickard and R. Needs, J. Phys. Condens. Matter 23, 053201 (2011).

[2] C. W. Glass, A. R. Oganov, and N. Hansen, Comput. Phys. Commun. 175, 713 (2006).

[3] C. E. Wilmer, M. Leaf, C. Y. Lee, O. K. Farha, B. G. Hauser, J. T. Hupp, and R. Q. Snurr, Nat. Chem. 4, 83 (2012).

[4] R. E. Schreiber, L. Houben, S. G. Wolf, G. Leitus, Z.-L. Lang, J. J. Carbó, J. M. Poblet, and R. Neumann, Nat. Chem. 9, 369 (2017).

[5] K. Himoto, M. Matsumoto, and H. Tanaka, Phys. Chem. Chem. Phys. 16, 5081 (2014).

[6] A. V. Radha, L. Lander, G. Rousse, J. M. Tarascon, and A. Navrotsky, J. Mater. Chem. A 3, 2601 (2015).

[7] T. Bartels-Rausch et al., Rev. Mod. Phys. 84, 885 (2012).

[8] J. C. Palmer, F. Martelli, Y. Liu, R. Car, A. Z. Panagiotopoulos, and P. G. Debenedetti, Nature (London) 510, 385 (2014).

[9] O. Mishima and H. E. Stanley, Nature (London) 396, 329 (1998).

[10] S. Klotz, T. Strässle, G. Hamel, R. J. Nelmes, J. S. Loveday, G. Rousse, B. Canny, J. C. Chervin, and A. M. Saitta, Phys. Rev. Lett. 94, 025506 (2005).

[11] J. Russo, F. Romano, and H. Tanaka, Nat. Mater. 13, 733 (2014).

[12] O. Mishima, L. Calvert, and E. Whalley, Nature (London) 310, 393 (1984).

[13] K. Amann-Winkel, R. Böhmer, F. Fujara, C. Gainaru, B. Geil, and T. Loerting, Rev. Mod. Phys. 88, 011002 (2016).

[14] E. B. Moore and V. Molinero, Nature (London) 479, 506 (2011).

[15] J. Espinosa, C. Navarro, E. Sanz, C. Valeriani, and C. Vega, J. Chem. Phys. 145, 211922 (2016).

[16] F. Pietrucci, Rev. Phys. 2, 32 (2017).
[17] W. Lechner and C. Dellago, J. Chem. Phys. 129, 114707 (2008).

[18] R. Martoňák, A. Laio, and M. Parrinello, Phys. Rev. Lett. 90, 075503 (2003).

[19] A. Haji-Akbari and P. G. Debenedetti, Proc. Natl. Acad. Sci. U.S.A. 112, 10582 (2015).

[20] M. Valle and A. R. Oganov, Acta Crystallogr. Sect. A 66, 507 (2010).

[21] G. A. Gallet and F. Pietrucci, J. Chem. Phys. 139, 074101 (2013).

[22] F. Pietrucci and R. Martoňák, J. Chem. Phys. 142, 104704 (2015).

[23] F. Pietrucci and A. M. Saitta, Proc. Natl. Acad. Sci. U.S.A. 112, 15030 (2015).

[24] L. Zhu et al., J. Chem. Phys. 144, 034203 (2016).

[25] S. De, A. P. Bartók, G. Csányi, and M. Ceriotti, Phys. Chem. Chem. Phys. 18, 13754 (2016).

[26] D. Branduardi, F. L. Gervasio, and M. Parrinello, J. Chem. Phys. 126, 054103 (2007).

[27] See Supplemental Material at http://link.aps.org/ supplemental/10.1103/PhysRevLett.119.245701 for computational details and additional data, which includes Refs. [28-37].

[28] H. J. Berendsen, J. v. Postma, W. F. van Gunsteren, A. DiNola, and J. Haak, J. Chem. Phys. 81, 3684 (1984).

[29] G. Bussi, D. Donadio, and M. Parrinello, J. Chem. Phys. 126, 014101 (2007).

[30] B. Roux, Comput. Phys. Commun. 91, 275 (1995).

[31] H. J. Berendsen, D. van der Spoel, and R. van Drunen, Comput. Phys. Commun. 91, 43 (1995).

[32] G. A. Tribello, M. Bonomi, D. Branduardi, C. Camilloni, and G. Bussi, Comput. Phys. Commun. 185, 604 (2014).

[33] M. Mendelev, S. Han, D. Srolovitz, G. Ackland, D. Sun, and M. Asta, Philos. Mag. 83, 3977 (2003).

[34] F. H. Stillinger and T. A. Weber, Phys. Rev. B 31, 5262 (1985).

[35] W. L. Jorgensen, D. S. Maxwell, and J. Tirado-Rives, J. Am. Chem. Soc. 118, 11225 (1996).

[36] A. P. Bartók, R. Kondor, and G. Csányi, Phys. Rev. B 87, 184115 (2013).

[37] J. Chiu, F. W. Starr, and N. Giovambattista, J. Chem. Phys. 139, 184504 (2013).

[38] A. Laio and M. Parrinello, Proc. Natl. Acad. Sci. U.S.A. 99, 12562 (2002)

[39] G. M. Torrie and J. P. Valleau, J. Comput. Phys. 23, 187 (1977).

[40] J. L. Abascal and C. Vega, J. Chem. Phys. 123, 234505 (2005).

[41] T. L. Malkin, B. J. Murray, A. V. Brukhno, J. Anwar, and C. G. Salzmann, Proc. Natl. Acad. Sci. U.S.A. 109, 1041 (2012).

[42] T. L. Malkin, B. J. Murray, C. G. Salzmann, V. Molinero, S. J. Pickering, and T. F. Whale, Phys. Chem. Chem. Phys. 17, 60 (2015).

[43] C. Vega, E. Sanz, J. Abascal, and E. Noya, J. Phys. Condens. Matter 20, 153101 (2008).

[44] J.-Y. Chen and C.-S. Yoo, Proc. Natl. Acad. Sci. U.S.A. 108, 7685 (2011). 